See discussions, stats, and author profiles for this publication at: https://www.researchgate.net/publication/331586169

\title{
El punto de vista y la construcción del objeto de estudio: "El sentido práctico en la elaboración de un estado del arte"
}

Method · March 2019

DOI: $10.13140 /$ RG.2.2.27608.14080

CITATIONS

0

1 author:

जा

Fabián Andrés Llano

Universitaria uniagustiniana

97 PUBLICATIONS 73 CITATIONS

SEE PROFILE

Some of the authors of this publication are also working on these related projects:

Project Enseñanza de la Gestión Urbana View project

Project Prácticas Pedagógicas y Didácticas View project
READS

545 


\section{El punto de vista y la construcción del objeto de estudio}

“El sentido práctico en la elaboración de un estado del arte”

Fabián Andrés Llano ${ }^{1}$

\section{Resumen:}

E1 siguiente texto pretende convertirse en un apoyo pedagógico para aquellos investigadores que busquen alternativas para la construcción de un estado del arte. Esta puesta en valor de una reflexión práctica sobre la elaboración de un objeto de estudio, retoma las reflexiones epistemológicas realizadas por Pierre Bourdieu en su obra el oficio del sociólogo y aporta al modelo de Adrián Serna Dimas sobre un diseño de investigación pospositivista. Estos aportes que se realizan se concretan en instrumentos de sistematización y elaboración textual como la ficha de sistematización de autores y un modelo de escritura científica y argumentativa para la elaboración de reseñas críticas.

Palabras clave: reflexividad, vigilancia epistemológica, objeto de estudio, campo de la producción, estados del arte

\footnotetext{
${ }^{1}$ Docente de la Universidad Piloto de Colombia Facultad de Arquitectura y Artes (ámbito currículo). Licenciado en Ciencias Sociales de la Universidad Distrital Francisco José de Caldas, Magíster en Investigación social interdisciplinaria de la misma Universidad y Candidato a Doctor en Ciencias Humanas del Patrimonio y la Cultura, Universidad de Girona (España). Investigador asociado Colciencias Mail: 1lanofabian@hotmail.com, ID 0000-0003-2181-3476.
} 


\section{I. la reflexión y la reflexividad}

"El punto de vista -dice Saussure-crea el objeto." Es decir que una ciencia no podría definirse por un sector de lo real que le correspondería como propio. Como lo señala Marx, "la totalidad concreta, como totalidad del pensamiento, como un concreto del pensamiento es, in fact, un producto del pensamiento y de la concepción [...]. El todo, tal como aparece en la mente, como todo del pensamiento, es un producto de la mente que piensa y que se apropia el mundo del único modo posible, modo que difiere de la apropiación de ese mundo en el arte, la religión, el espíritu práctico. El sujeto real mantiene, antes como después, su autonomía fuera de la mente [...]" $[1]$ [K. Marx, texto $n^{\circ}$ 20]. Es el mismo principio epistemológico, instrumento de la ruptura con el realismo ingenuo, que formula Max Weber: "No son-dice Max Weber- las relaciones reales entre "cosas» lo que constituye el principio de delimitación de los diferentes campos científicos sino las relaciones conceptuales entre problemas. Sólo alli donde se aplica un método nuevo a nuevos problemas y donde, por lo tanto, se descubren nuevas perspectivas nace una «ciencia» nueva" [2] [Max Weber, texto $n^{o}$ 21].

(Bourdieu, 2002)

La anterior cita, aunque extensa, resume de manera contundente la renuncia a las formas netamente empiristas en la elaboración de un estado del arte. Esta mirada rompe con la idea de la elaboración de un estado del arte a partir de una sumatoria de resúmenes analíticos. También se distancia de la idea recurrente de confundir estados del arte con estudios de corte bibliométricos, que no hacen más que exaltar la legitimidad que han obtenido algunos buscadores o bases de datos como Scopus. Más allá de mirar únicamente los resúmenes de las investigaciones y guiarse por palabras claves, la elaboración de un estado del arte requiere promover la recuperación de los diseños investigativos y los resultados de investigación, pero lo que se hace más urgente es distanciarse de la idea recurrente de referenciar únicamente artículos posicionados en estos repositorios antes mencionados, que valga decir no son de acceso abierto, y que buscan lucrarse con el esfuerzo y la dedicación de investigadores que buscan posicionarse en el mundo académico ${ }^{2}$.

\footnotetext{
${ }^{2}$ De acuerdo con Guedón (2011) las divisiones entre centro y periferia en la producción científica se hacen más evidentes con el negocio lucrativo que supone la industria editorial. Hoy en día, la Web de la Ciencia (Web of Science), actual encarnación online del SCI, ofrece herramientas para evaluar no sólo revistas ("Informes de Citación de Revistas", "Journal Citation Reports"), sino que, incluso, permite realizar un seguimiento de los autores más citados. Los países están clasificados rutinariamente y las instituciones se encuentran en una especie de cuadro de honor. La lista de posibilidades aparece en los "Indicadores Básicos de la Ciencia" ("Essential Science Indicators") relacionados con la Web de la Ciencia. Ahora bien, aunque esto sigue sucediendo, también aparece la posibilidad de un acceso más abierto que equiparan un poco más la balanza. Una revista de acceso abierto, procedente de cualquier sitio, posee mejores posibilidades de ser utilizada en todas partes que una revista de acceso cerrado, y el creciente poder de los diversos motores de búsqueda incrementará las probabilidades en este sentido: Google (y en particular, Google Académico), Yahoo! y otros poderosos motores
} 
Para no entrar en el debate sobre la necesidad del acceso abierto en la producción, circulación y consumo de conocimiento que contribuya con el conocimiento mutuo, parece acertada la recomendación del sociólogo Pierre Bourdieu de basar, un plan para una ciencia de las relaciones internacionales en materia de cultura, en el reconocimiento de los mecanismos sociales presentes en la construcción de conocimiento. De esta manera, las posibilidades de realizar historias sociales comparadas, la búsqueda de homologías de posición, crear las condiciones sociales de un diálogo racional, el reconocimiento del conocimiento científico de los campos de producción nacionales, darían como resultado unas historias sociales reflexivas y criticas dedicadas a actualizar las estructuras del inconsciente cultural nacional mediante un socio análisis científico (Bourdieu, 2002).

Las preguntas que aparecen después de esta pequeña reflexión son las siguientes: ¿de qué manera se pueden realizar estos balances y sobre qué criterios se puede sostener un diálogo racional que contribuya a quebrar las relaciones entre el centro y la periferia en la producción de conocimiento? ¿cuál es el papel que cumplen la elaboración de estados del arte en estos debates? Específicamente frente a la construcción de objetos de estudio ¿cuál es la necesidad de realizar un número específico de referencias de estas bases de datos antes mencionadas si esto no va a permitir tomar decisiones sobre el diseño de la investigación? Y unas preguntas mucho más directas y oportunas ¿de qué manera se puede realizar un estado del arte teniendo en cuenta las reflexiones antes mencionadas? ¿cuál es el sentido práctico de en la construcción de objetos de estudio? Con seguridad, frente a las primeras preguntas las respuestas requieren esfuerzos colectivos y disquisiciones epistemológicas que rebasan el propósito de este texto. Por esta razón, el texto centrará su atención en ofrecer una respuesta práctica frente a las dos últimas preguntas planteadas.

Un estado del arte, según las lecturas realizadas por el propio Bourdieu, no es ni más ni menos que la propia construcción del objeto de estudio. En este orden de ideas una de las primeras tareas a realizar es sin duda darle mayor sentido y practicidad a la construcción del objeto de estudio en la investigación social de segundo orden. Bajo un ejercicio de reflexividad que invita a una vigilancia epistemológica en la construcción del objeto de estudio, el siguiente texto se centra en los planteamientos procedimentales para la construcción de un estado del arte. Cabe anotar que, no siendo la única forma de llevar a cabo operaciones intelectuales conducentes a la construcción de un objeto de estudio, es procedente en cuanto a la pertinencia y unas formas mucho más delimitadas de abordaje conceptual a propósito de intereses y posturas epistémicas y epistemológicas particulares y particularizadas. Estas posturas armonizadas en tanto líneas de investigación, intereses investigativos y diferentes regímenes representacionales, se convierten en el punto de vista

de búsqueda, en efecto, comienzan a nivelar el campo de juego que las bibliografías de alto costo (como el SCI) torcieron. (Guedon, 2011, 163-165 
sobre el cual gira la indagación de referentes conceptuales, teóricos y metodológicos. Si bien estos ejercicios de conceptualización aportan a las líneas de investigación de los diferentes programas, este documento pretende dar soporte epistemológico, teórico, metodológico a diferentes propuestas de revisión documental que requieran aclarar algunas de las operaciones más relevantes en la construcción del estado del arte.

\section{Estrategia ideada para la construcción de estados del arte}

La siguiente propuesta retoma las ideas de Adrián Serna Dimas en el procedimiento de elaboración del estado del arte. Estas recomendaciones pueden consultarse en extenso en el libro Disertación elemental algunas cuestiones sobre la investigación social, editado en el año 2015 por la Universidad Santo Tomás. Además, de darle un sentido más práctico a las reflexiones de Pierre Bourdieu sobre la construcción del objeto y a la propuesta de Serna en la concreción de las reflexiones bourdivianas, este texto se propone desarrollar unos instrumentos que ayuden al proceso de producción textual del estado del arte. En este sentido, se proponen algunas cuestiones relacionadas con la operacionalización de esta importante elaboración conceptual, que complementan de alguna forma, la propuesta del sociólogo Pierre Bourdieu y del antropólogo Adrián Serna Dimas.

Así las cosas, el planteamiento de una metodología para la elaboración de un estado del arte procede bajo un ejercicio sistemático que incluye varias consideraciones a saber: en primer lugar, que la construcción de un estado del arte obedece a unos intereses investigativos particulares y en ocasiones a unas intencionalidades institucionales que proyectan la investigación desde sus líneas de investigación o desde unos derroteros, enfoques o temas particulares. Estas intencionalidades investigativas se concretan en un punto de vista, sometido al debate con posturas académicas y científicas cercanas al régimen representacional propuesto para la indagación.

En segundo lugar, que más que una búsqueda de referencias sobre un tema en particular, el estado del arte logra afianzar estos intereses en relación con una problemática particular inscrita en los enfoques, tendencias e incluso paradigmas circunscritos en las temáticas o intereses concretos, representados en el punto de vista y en la enunciación propuesta. En tercer lugar, que el planteamiento de un estado del arte se opone para este caso, a las lógicas de construcción de antecedentes donde impera una lógica del enciclopedismo anclada a las prácticas de indagación del siglo XVIII y que valga decir, ha sido domesticada por el positivismo y proyectada como un referente investigativo en las prácticas investigativas contemporáneas como los escenarios académicos donde se encuentran por supuesto las universidades. En cuarto lugar, más que buscar afianzar un objeto de estudio dado, lo que plantea la lógica del estado del arte es la construcción del objeto de estudio desde la relación entre el sujeto que investiga y el escenario y campo problemático desde el cuál se aborda la indagación. 


\section{Operaciones del estado del arte}

Al partir de unos intereses concretos, el estado del arte busca afianzar un punto de vista en relación a una problemática en un contexto particular. En este orden de ideas todo objeto de estudio que se pretenda construir hace parte de un régimen representacional o una práctica situada que expone de antemano unos mecanismos argumentativos, que son considerados en tanto expresan una postura epistemológica, una tesis, un marco teórico, un marco metodológico y unos resultados de investigación.

Por poner un ejemplo, desde un enfoque construccionista, la realidad no está dada, sino que obedece a las tensiones e interacciones de diferentes agentes y agencias que participan en el proceso de construcción de la realidad. Desde este ángulo epistemológico, la realidad procede bajo interacciones, relaciones y manifestaciones simbólicas de unas representaciones sobre la realidad, que valga decir, a su vez proceden de otras las representaciones de la realidad que han intentado objetivar el mundo social. En esta medida en el estado del arte se requiere una vigilancia epistemológica mayor frente a estos metalenguajes que tienen de por si elementos conceptuales y metodológicos y unas condiciones de producción particulares. Por tal motivo, en la lógica del estado del arte, se logra mayor disquisición epistemológica bajo un ejercicio de reflexividad ${ }^{3}$.

De acuerdo con Serna (2015) desde una práctica investigativa reflexionada, se pretende superar la distinción entre el objeto existente en la realidad y el objeto como producto de la intelección. Estas relaciones entre lo dado y lo conocido y entre la realidad y la representación, requieren implementar en la práctica una profundización conceptual e incluso una rehistorización conceptual, con el propósito de vislumbrar los diferentes lugares de enunciación. En este sentido, el estado del arte busca dar cuenta de cómo unos autores desde unos campos especializados abordan problemáticas investigativas similares al objeto de estudio que se pretende construir. Estas operaciones según Serna (2016) se realizan bajo el presupuesto que dichos autores no investigan en limpio, sino que interponen unos diseños de investigación que, si bien involucran, preguntas, objetivos e hipótesis, también ponen en

\footnotetext{
${ }^{3}$ Admitir la reflexividad del mundo social tiene varios efectos en la investigación social. Primero, los relatos del investigador son comunicaciones intencionales que describen rasgos de una situación, pero estas comunicaciones no son "meras" descripciones, sino que producen las mismas situaciones que describen. Segundo, los fundamentos epistemológicos de la ciencia no son independientes ni contrarios a los fundamentos epistemológicos del sentido común que operan sobre la misma lógica. Tercero, los métodos de la investigación social son básicamente los mismos que los que se usan en la vida cotidiana. Es tarea del investigador aprehender las formas en que los sujetos de estudio producen e interpretan su realidad para aprehender sus métodos de investigación. (Guber, 2001).(PDF) (Llano, 2010). El sujeto objetivante en la investigación social. Available from: https://www.researchgate.net/publication/321106836 El sujeto objetivante en la investigacion social [accessed Mar 06 2019].
} 
discusión la pertinencia de unos sistemas teóricos y metodológicos. Es en la profundización conceptual donde es procedente definir tendencias, enfoques e incluso paradigmas.

Visto de esta manera, esta propuesta procedimental de construcción de un estado del arte, involucra las siguientes preguntas: ¿Desde qué lugar de enunciación o régimen representacional está participando el autor consultado? ¿De qué manera realizó el planteamiento del problema? ¿cuáles fueron los conceptos y metodologías utilizadas por el autor? ¿Se establece coherencia entre el planteamiento del problema, el enfoque epistemológico y el sistema conceptual y metodológico propuesto? Desde estos interrogantes se establece una discusión entre los autores consultados y el punto de vista sobre el cual se está realizando la indagación; esto permite un fortalecimiento argumentativo del propio punto de vista y una depuración de las mismas dificultades presentadas que se resolverán en la producción investigativa.

ORGANIZACIÓN DE LOS AUTORES

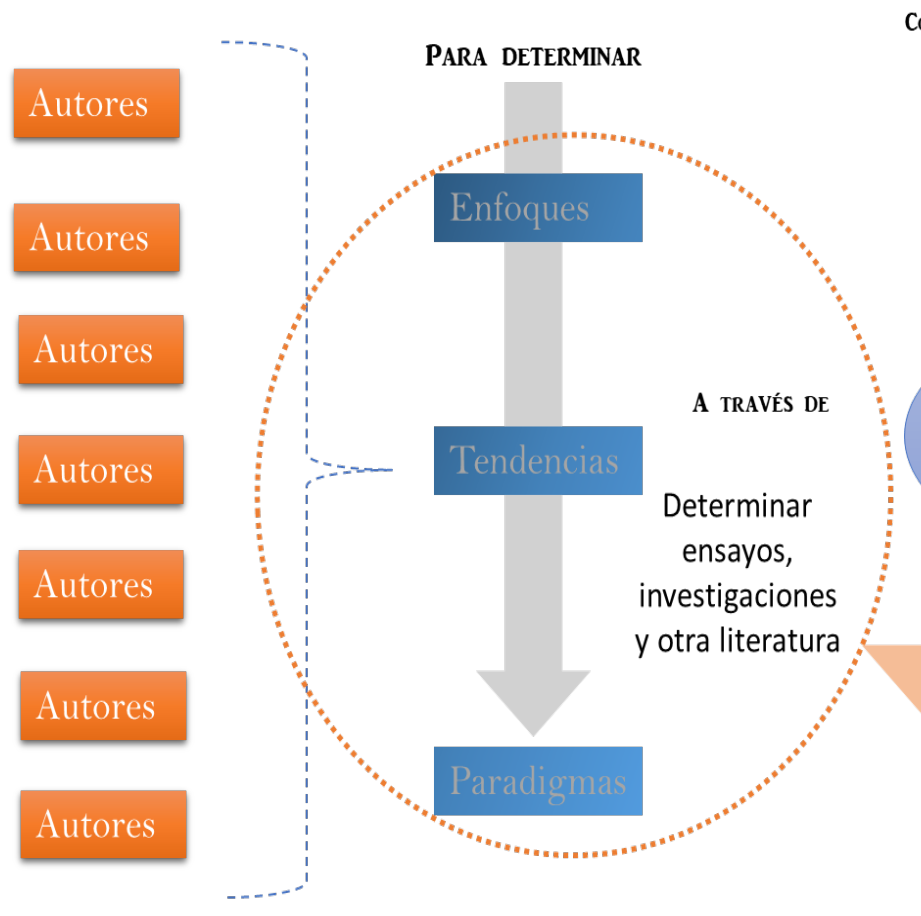

ORGANIZAR LA DISCUSIÓN POR CATEGoRÍAS
CON EL FIN DE DETERMINAR LOS

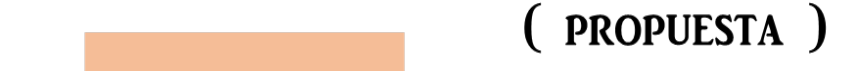

CONSTRUCCIÓN DE ANTECEDENTES

Problemas más notables

Principales discusiones

Teorías y metodologías

Más utilizadas para resolver

Investigaciones

figura 1 (Gráficos elaborados por el autor) 


\section{Organización conceptual}

En estos términos un estado del arte procede no solo como el mecanismo para la construcción del objeto de estudio, sino además conduce a la proyección investigativa de un punto de vista objetivado que pone en juego, la argumentación de fortalezas y debilidades de una práctica situada. Esta práctica investigativa o un régimen representacional especifico que procede mediante un habitus académico requiere ser sometido a sometido a critica. Desde los planteamientos de Serna (2016) el abordaje del estado del arte procede bajo la construcción de ejes conceptuales que, si bien son provisionales, pueden circunscribir la discusión en torno a una problemática, un contexto y un punto de vista (enunciado investigativo, régimen representacional, postura epistémica o epistemológica). De este modo, se pasa a definir los ejes conceptuales teniendo en cuenta los intereses del investigador o los investigadores y en la mayoría de los casos, los intereses de la institución en cuanto a líneas de investigación.

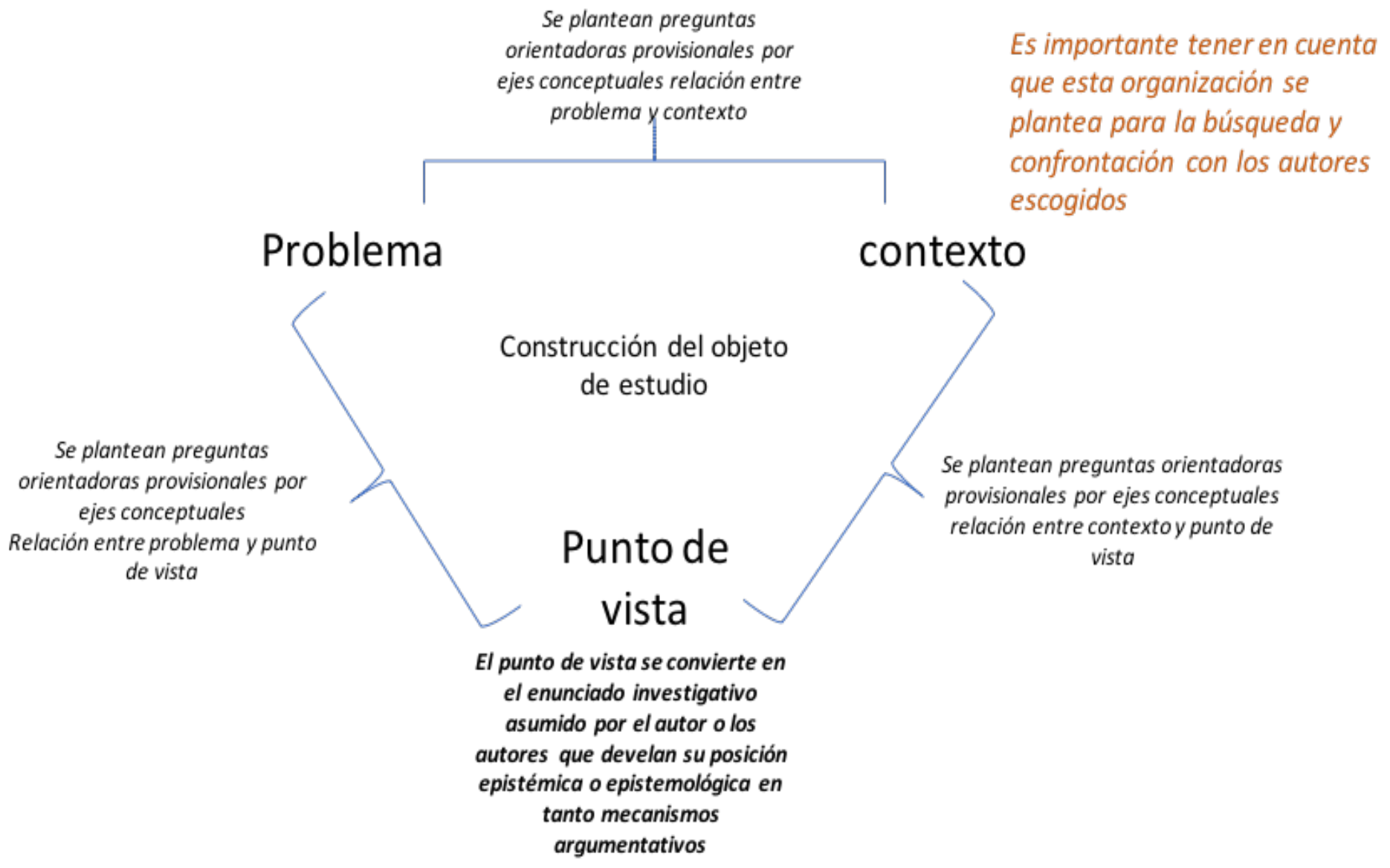

Fuente: elaboración propia

Una vez se plantee el esquema que asegura una delimitación de la búsqueda de autores por ejes conceptuales, se pasa en efecto, a la indagación de propuestas investigativas relevantes en tanto portadoras de mecanismos argumentativos. Las preguntas orientadoras permitirán 
decantar una mejor búsqueda y centrar la discusión en relación con las intuiciones apenas creadas desde la construcción de un enunciado investigativo que se convierte en los mecanismos argumentativos con los cuales se aborda una búsqueda. Mediante un pensamiento por comprensión y contrastación, las propuestas investigativas se centrarán en buscar unos autores en particular bajo el criterio de buscar en lo poco de manera intensa. Una vez realizada la busqueda se pasa a la lectura y la extracción de mecanismos argumentativos(Ver ficha de sistematizacion de autores). Como tercer procedimiento se pasa a la elaboración de reseñas criticas para evidenciar las tendencias, los enfoque o los paradigmas en la discusión objetivada. Esta última parte tendrá un soporte importante en la segunda parte de este texto dedicada a las instrumentos.

El estado del arte desde el Positivismo

\section{El estado del arte desde Pospositivismo} El objeto de investigación es una transparencia
acotada tanto de un problema humano o social
como de un problema de investigación, es decir,
el objeto es un derivado esencial de la
caracterización, la delimitación y la justificación
que realiza el sujeto, pero acatando el discurrir
natural de la realidad.
El estado del arte supone la ubicación de un conjunto de tradiciones o tendencias que, desde autores y obras específicas, realizaron investigaciones desde problemas, contextos o puntos de vista iguales o semejantes a los del planteamiento del problema. En este sentido, el estado del arte busca la construcción de un campo de puntos de vista posibles que confronten, contrasten y enriquezcan las conexiones entre problema, contexto y enunciado. El estado del arte no admite ninguna caracterización que prescinda de autores y obras.

Fuente: Serna (2015)

La intención de someter el enunciado investigativo a las consideraciones de otros autores, además de evidenciar un repertorio de mecanismos argumentativos, está atada precisamente en la comprensión de las relaciones y las interacciones que se establecen entre unos cuerpos epistemológicos, disciplinares y problemáticos en el cual se inscribe el problema de investigación. La construcción de un objeto de estudio no puede estar ajeno a las elecciones epistemológicas, conceptuales, metodológicas y hasta formativas que pone en discusión un investigador mediante el estudio. Tal y como plantearía Ortega y Gasset en su ensayo sobre 
el estudiar y el estudiante de 1993, el mismo acto de estudiar promueve un compromiso mayor con la apropiación de la cultura, comprendida aquí como la posibilidad de saber más sobre la disciplina y las posibles conexiones con otros regímenes representacionales

De este modo, estudiar las relaciones y los campos problémicos propuestos en la construcción del estado del arte, se convierte en una condición necesaria para tender puentes (entender) entre unas preconcepciones y los dominios teóricos, metodológicos y conceptuales de disciplinas y relaciones interdisciplinares cambiantes. Habría que advertir, que, sin llegar a ser del todo abarcador, estos objetos de estudio argumentados, nunca estandarizados, sino cambiantes, forman parte del universo de la disciplina (Fourez, 1998). Estos objetos de estudio, que potencian las necesidades de saber en el investigador en correspondencia con una apropiación progresiva de la disciplina, se convierten en la puerta de entrada a la estructura de los campos de la producción de la ciencia.

Finalmente, al tener presente que, desde la práctica investigativa, las descripciones y las observaciones de la realidad no proceden únicamente bajo el racero empírico de un investigador desprevenido, la construcción de los objetos de estudio proyecta la discusión por unos aparatos teóricos y metodológicos pertinentes para la construcción de lenguajes y representaciones sobre la realidad a objetivar. De esta manera los diferentes problemas de investigación propuestos inicialmente desde la construcción de un objeto de estudio se proyectan a la toma de decisiones en lo teórico y lo metodológico. El estado del arte es la posibilidad de reconocer, más allá de las estructuras universitarias, las posibilidades y la amplitud de los desarrollos disciplinares de unas tradiciones conformadas por comunidades académicas, redes de conocimiento, procesos de comunicación y lenguajes teóricos y metodológicos, además del reconocimiento de diferentes prácticas y modos de investigación. 


\section{Los instrumentos}

Una de las formas de llevar a cabo este proceso de construcción de un estado del arte es a través de unos instrumentos claves que permitan concretar en la práctica estas intencionalidades. Por una parte, aparece, la ficha de sistematización de autores que busca convertirse en el instrumento donde pueden quedar consignados los principales mecanismos argumentativos de los autores una vez leídos. Más que leer, resulta importante releer y comprender las intencionalidades de los autores consultados.

\section{La ficha de sistematización de autores}

Esta ficha es un instrumento sencillo que puede ser utilizado una vez se tengan los ejes conceptuales provisionales y una primera revisión bibliográfica realizada. Aquí es importante tener en cuenta que la lectura sistemática de los autores escogidos, será la clave para una elaboración rigurosa de estos del arte. Una primera recomendación a la hora de abordar la lectura es que esta se realice por medio de un sistema de preguntas como las siguientes: ¿Cuál es la tesis que plantea el autor? ¿cuál fue el marco teórico y metodológico utilizado por el autor? ¿qué posición epistemológica encarna con sus decisiones teóricas y metodológicas? En últimas se puede asumir la propuesta realizada por Julia Baquero y Felipe pardo en cuanto a la lectura de un texto científico y argumentativo. Sin un sistema de preguntas que pueda desentrañar las estructuras argumentativas subyacentes en el mismo proceso de construcción de los textos científicos y sin la identificación de los diseños y los procesos de investigación llevados a cabo por parte del investigador, es poco probable que se logre extraer unos mecanismos argumentativos solventes para elevar la discusión sobre la construcción de un objeto de estudio. 
Bayuero julin a Felipe Purdo

Textos crenticies y cromantitivos una metuloluoia pura su compren. $\sin 4$ relućcion

1 Murco Refirencial
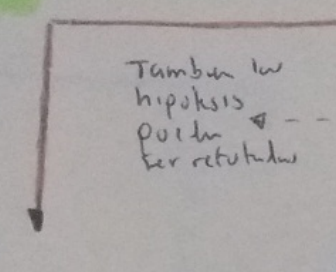

2 Objelo especitico

3 Probumu

4 mitulo especitios

5 soluciu

6 (anclusione)

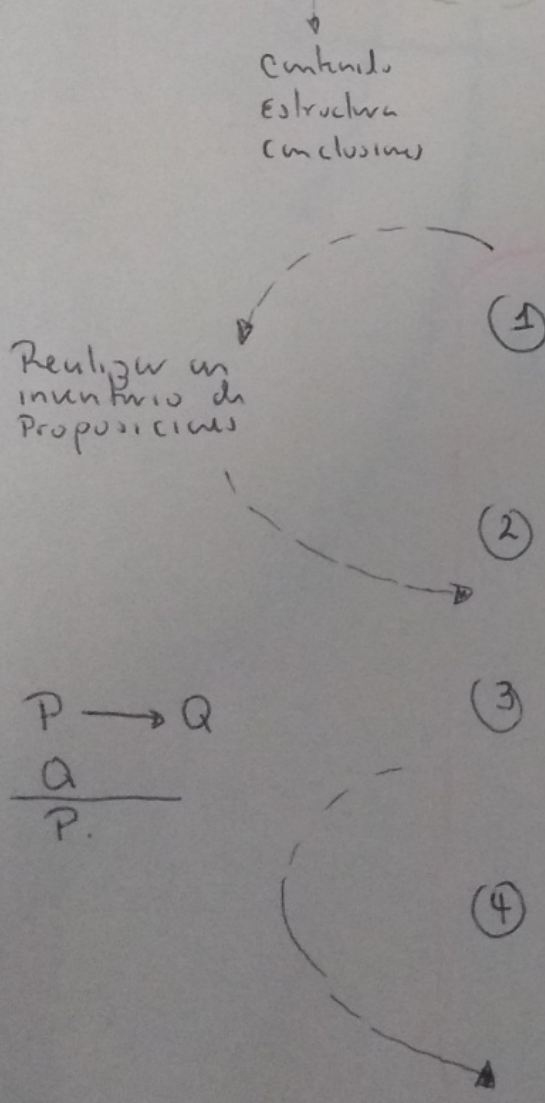

\section{Instumito}

ch lecturny reluccion

sistima is cunceph

e hipofesis y hechos

que dom ouruntia en

valiliz a la premise

mayur he los raciocinas involucrados en los proctios

$\$ 1.1$ subir 0 disciplina 4 so objeto

1.2 Lus tareas al suber 0 disciplian-

1. 3 critirios de ahivacion Cepishomologito)

1.4 \&ा Murco conceptual 1.4 .1 El Muco tioriss

1. 2 . Lus culdicion muinds

1. 5 El murco metodulogiró

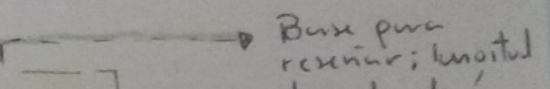

Hipotesis de

leclura pa

parte di

lectur.<smiles>[Hg]C1CC1</smiles>

$\int$

Aspecilos sustutives

y metuloluciess

in la invétioncio resenums: inoritus cheuch is

(1) Destinator.s.

(2) objetivo hil inturn

(3) Espucis y times dusponibu.

Buscer lus funcomes de las proposicions su relucion hipotess-sostantur cin

(2) Estublecer Ins conexims logirs-eviencials entre dichus proposilimes

3) Purn culn conexic, re(n) truiv el raciocinio tipo reductivo que sirve $h$ bux

(4) Purn in premisa munar de cula raciocincos, ca buxe en al texto imh her la ky y hecho ye permiton lu comeric entre cintececute y cmx $\frac{P \rightarrow Q}{P}=\underset{\substack{\text { dulo } \\ \text { que... }}}{\text { Hurvinul. }}$ cuenk ... dudo yue...

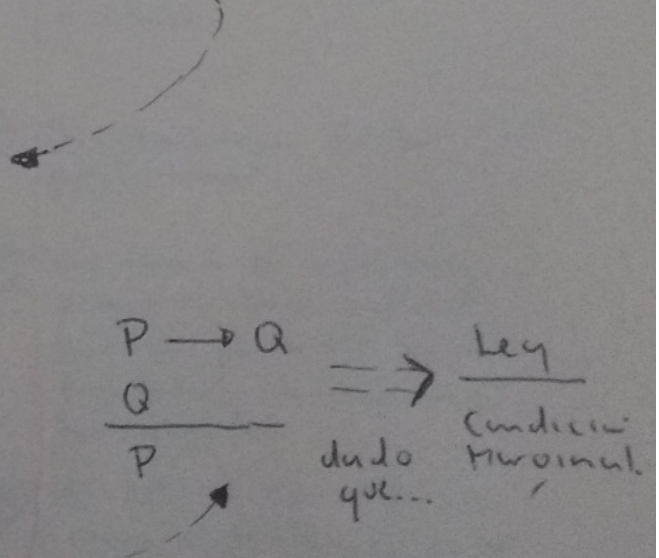


Nombre del Proyecto:(titulo de la investigación y preguntas )

El autor, El titulo del texto, año de publicación

Eje conceptual al cual le aporta:

\begin{tabular}{|c|c|c|c|c|}
\hline $\begin{array}{c}\text { Cómo se relaciona lo que } \\
\text { dice el autor con mis } \\
\text { preguntas. } \\
\text { tesis autores Vs } \\
\text { enunciado. }\end{array}$ & $\begin{array}{c}\text { Qué } \\
\text { CONCEPTOS } \\
\text { utiliza. el } \\
\text { autor } \\
\text { Enúncielos }\end{array}$ & $\begin{array}{c}\text { Extraer del texto el marco teórico presentado } \\
\text { por el autor consultado y además de } \\
\text { identificarlo es importante sistematizar la } \\
\text { forma en que aplicó ese marco teórico desde } \\
\text { la propuesta de investigación. Esa es la } \\
\text { pregunta por el cómo }\end{array}$ & $\begin{array}{c}\text { Buscar las justificaciones que } \\
\text { presenta el autor en tanto } \\
\text { aplicaciones teóricas y } \\
\text { metodológicas. Es la pregunta } \\
\text { por el por qué }\end{array}$ & $\begin{array}{c}\text { Buscar las consideraciones } \\
\text { prácticas de la }\end{array}$ \\
$\begin{array}{l}\text { investigación en cuanto a la } \\
\text { solución de problemas } \\
\text { específicos. Esa es la } \\
\text { pregunta por el para qué }\end{array}$ \\
\hline
\end{tabular}

OPINIÓN CRITICA: una vez se tengan los mecanismos argumentativos del autor representado en las tesis(Qué) las teorías y las metodologías (el cómo y el por qué) y los resultados (el para qué) se pasa a tomar postura crítica frente al texto. ¿Este texto se relaciona con lo que usted está indagando? ¿de qué manera le aporta?, ¿De qué manera no le aporta? ¿cuáles son los aciertos? ¿Cuáles son las fallas? 


\title{
La reseña critica: un punto de vista objetivado
}

\begin{abstract}
Quisiera expresar solamente algunas ideas deshilvanadas que se me ocurrieron mientras oía hablar sobre Foucault. En reuniones como ésta es importante saber que el problema está precisamente en eso de lo que se habla; de modo que siempre es necesario someter las citas a la crítica, examinar su función, su verdad, su validez. Se puede oponer una cita de Foucault a otra no solo porque se contradijo, como todo el mundo, sino también porque no dijo la misma cosa en el mismo momento o a las mismas personas, según las circunstancias (lo que no quiere decir que mintió aquí o allá). (Bourdieu, 2008, p13, 14)
\end{abstract}

La cita que se propone para introducir el tema de la reseña crítica, es sin duda, una forma de evidenciar los problemas que se tienen a la hora de escribir o comentar alguna actividad académica. No solo basta con interpretar al autor, sino ponerlo en el contexto de su acto de habla donde, no solo pone en juego las mismas competencias lingüísticas, sino lo que él o ella representa como punto de vista. Hacer hablar a un autor, significa, ante todo, someter el punto de vista que representa a las condiciones de la producción de estos discursos. En el caso de las reseñas críticas, significaría poner de presente el punto de vista que representa cada una de las posiciones que hacen parte de la construcción del objeto de estudio

El siguiente apartado se constituye en una guía académica cuyo propósito es fortalecer las argumentaciones y los diferentes puntos de vista a la hora de concretar la construcción del objeto de estudio en lo que se denomina estados del arte. Este documento-guía, pretende orientar la forma de elaboración de las reseñas críticas en función de una elaboración documental sólida con posibilidades de convertirse en los insumos básicos de la construcción de estados del arte. Este documento tiene tres partes. En la primera de ellas, se asume un escenario de comprensión de la reseña crítica. En la segunda parte, se propone un acercamiento a la elaboración de argumentos en tres momentos, que para el caso de la reseña crítica se convierten en las partes que componen dicho documento. En estos momentos de elaboración se presentan algunos ejemplos de cómo construir los argumentos desde un trabajo académico riguroso y esquemático para lograr objetivar de manera categorial, los principales argumentos en forma de tesis y argumentos que sostienen estas tesis (pensados para este caso en las razones para creer que la tesis es verdadera), en asocio a una lógica de evidencias (lógico evidencial).

Finalmente, para aquellos que quieran o deseen profundizar en estas formas de comprensión de los textos científicos y argumentativos, en la tercera parte se plantean unas referencias disponibles en la red. En dado caso de no comprender el ejercicio, se plantea un 
escenario mucho más vivencial y experiencial de la aplicación de estas formas de lectura y escritura propuestas en este pequeño documento.

\section{¿Cómo se comprende?}

La reseña critica se comprende como una lectura que hace un investigador en forma de escrito sobre los principales momentos del proceso de investigación de un autor. En este sentido, es la puesta en valor de un punto de vista que intenta, rescatar de forma categorial, los principales momentos de la investigación de cada uno de los autores escogidos para la elaboración del estado del arte. Es un texto de carácter académico que difícilmente se reduce a un resumen. En efecto, se distancia del resumen, en la medida en que logra rescatar la tesis y los argumentos más sobresalientes de los autores consultados bajo un hilo argumentativo sólido. Más ligado al análisis y a la síntesis, la reseña crítica se convierte en un punto de vista objetivado que es el resultado de una comprensión e interpretación de la investigación de un autor.

\section{¿Cómo se realiza?}

Como posibilidad de un análisis y una síntesis de las diferentes posturas en la construcción de un estado del arte, la reseña crítica se convierte en un mecanismo poderoso de sistematización. Estas formas de objetivación están ligadas a las diferentes posibilidades en la toma de decisiones, en la medida, en que, como insumo, la reseña crítica se convierte en un referente de apoyo de la investigación.

\section{Tres momentos clave para su realización}

Antes de presentar una de las formas como se puede realizar una reseña crítica, cabe mencionar que este análisis y síntesis que el investigador realiza, entra en correspondencia con la posibilidad de agrupar, disociar y categorizar los diferentes puntos de vista presentados en la investigación de un autor.

\section{Primer momento: identificar las principales tesis de la investigación}

Este primer momento es de suma importancia, en la medida en que el investigador busca resaltar las principales posturas de los autores, no desde, las anécdotas y los lugares comunes, sino desde su potencial argumentativo. Para lo cual se hace necesario objetivar con la asociación de ideas y el análisis del investigador, la búsqueda de los principales argumentos que sostienen esas tesis.

\section{Ejercicio}


La identificación de las principales tesis de un texto se puede lograr al rastrear, por medio de la producción de un "inventario de proposiciones", las proposiciones más importantes que definitivamente no pueden ser suprimidas del texto. Para tener claridad sobre este asunto, es importante comprender las diferencias entre oraciones y proposiciones:

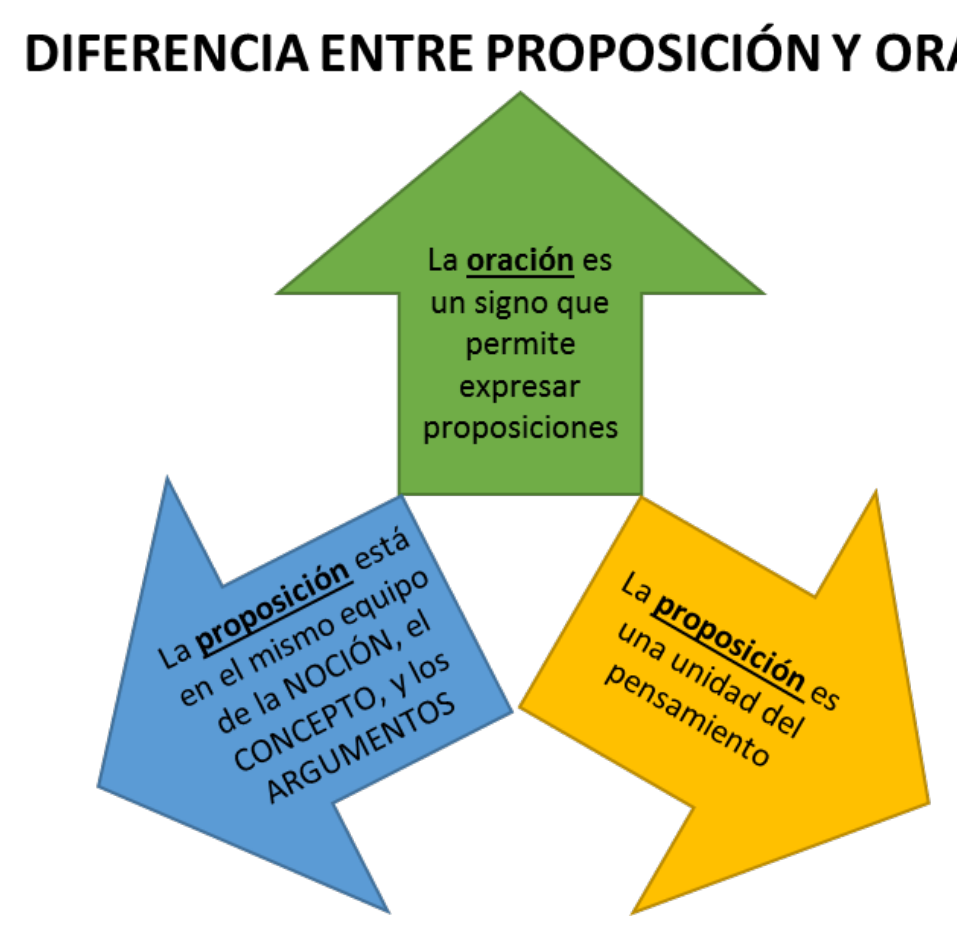

Fuente: Elaboración propia a partir de las referencias de Julia Baquero y Felipe Pardo

\section{Ejercicio práctico con un párrafo}

Se toma el texto y en el ejercicio de lectura se ubican las proposiciones. Por lo menos de punto a punto se encuentra una proposición. La idea es reconvertir la oración en proposición. Ejemplo:

\begin{tabular}{ll|l}
\multicolumn{4}{c}{ Párrafo literal } & \multicolumn{2}{c}{ Inventario de proposiciones } \\
\hline La emergencia y evolución como campo & 1. La evolución de la pedagogía como \\
disciplinar del saber pedagógico es paralelo al & campo disciplinar es paralelo a las \\
desarrollo de los sistemas públicos de enseñanza, & reformas escolares y los sistemas \\
particularmente en interacción con las reformas & semipúblicos. \\
escolares. La denominación francesa de & \\
"Ciencias de la Educación", al tiempo que una &
\end{tabular}


revalorización cientifica de su estatus, expresa también la disolución del saber pedagógico que la modernidad (desde Comenio a Durkheim, pasando por Kant y tantos otros), hizo de la educación. La pedagogía se dispersa, pues, en un conjunto de saberes, por un lado, que reclaman su propio estatus disciplinar; por otro, pretende ver reconocido en la Academia dicho carácter científico mediante la aplicación de disciplinas ya constituidas.
2. La disolución de la pedagogía en la modernidad revalorizó el saber pedagógico con la emergencia de las ciencias de la educación en Francia.

3. Con la modernidad la pedagogía se dispersa en un conjunto de saberes que reclaman autonomía disciplinar.

\section{Preguntas:}

¿Cuál de las tres proposiciones es la tesis? Si las tres proposiciones resultan importantes, entonces se hace necesario proponer una cuarta:

4. En la evolución de la pedagogía como campo disciplinar se presentó la dispersión de diferentes cuerpos de saberes que reclamaron su autonomía.

\section{Segundo momento: relacionar las tesis con las razones para creer en estas posturas}

En este segundo momento, se hacen evidentes las argumentaciones de las diferentes posturas encontradas. Aquí cabe mencionar que frente a un mismo argumento pueden girar varias posturas, que pueden ser conciliadas en relación a las tesis y los argumentos. La recomendación aquí, es que si se logran evidenciar estas macro-proposiciones (tesis, grandes temas), se puede también rescatar la secuencia proposicional de cada una de las tesis quedando organizado el desarrollo del texto en grandes bloques argumentativos respondiendo a la tesis (Qué) y a los mecanismos argumentativos el (¿Cómo?), el (¿por qué?) y el (¿Para qué?).

Una vez se cuenta con el inventario de proposiciones se pasa a la jerarquización de las mismas, en función de su articulación con la tesis o las tesis encontradas. De esta manera, se elabora un gráfico o una red argumentativa que permite ver con claridad la función que cumple cada una de las proposiciones en la argumentación.

\section{Ejercicio práctico}

1. La evolución de la pedagogía como campo disciplinar es paralelo a las reformas escolares y los sistemas semipúblicos. 
2. La disolución de la pedagogía en la modernidad revalorizó el saber pedagógico con la emergencia de las ciencias de la educación en Francia.

3. Con la modernidad la pedagogía se dispersa en un conjunto de saberes que reclaman autonomía disciplinar.

4. En la evolución de la pedagogía como campo disciplinar se presentó la dispersión de diferentes cuerpos de saberes que reclamaron su autonomía.

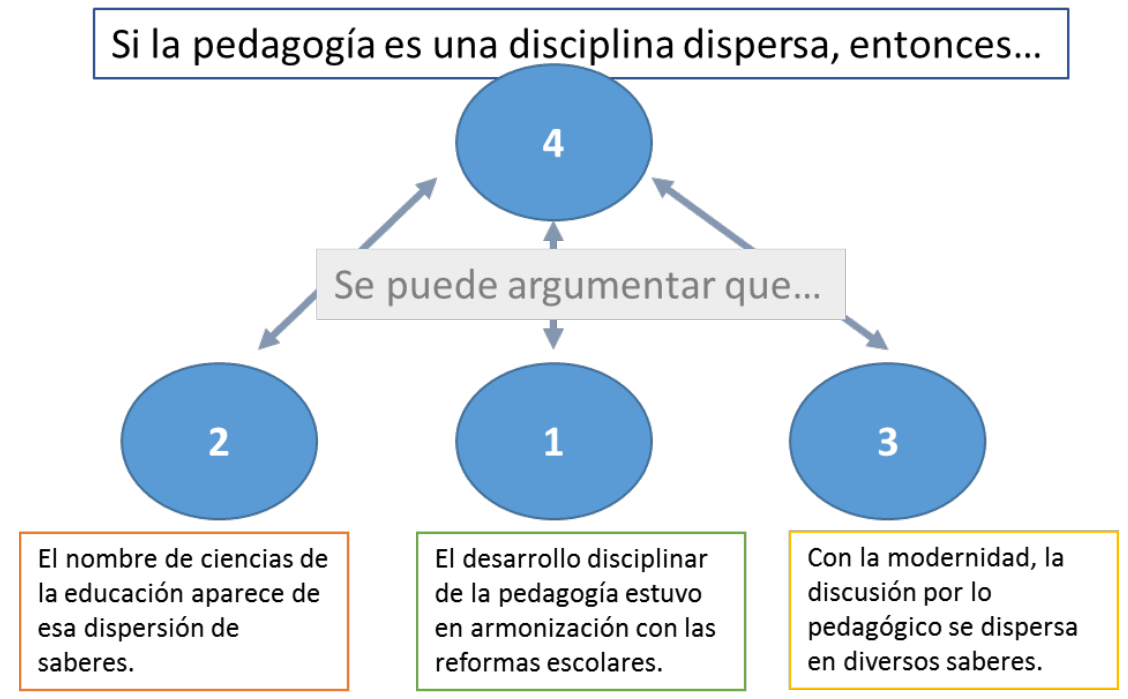

Con estas relaciones entre la tesis y las razones para creer que es verdad, se completa la estructura argumentativa rescatada de los diferentes textos (escritos, relatos, etc..) en formas sólidas de argumentación. En este sentido, la argumentación quedaría de la siguiente manera:

"La pedagogía es una disciplina dispersa. Las razones para pensar en esta dispersión se encuentran amparadas en tres razones fundamentales. En primer lugar, con la modernidad el saber pedagógico queda sometido a diferentes discusiones que más que unificar criterios frente a su estatuto disciplinar, dispersó la discusión por su objeto desde diferentes perspectivas que abrieron las posibilidades de conformación de nuevos saberes. En segundo lugar, la discusión por lo pedagógico tuvo como correlato las reformas escolares, con lo cual se establece el caldo de cultivo para la aparición de las denominadas ciencias de la educación. Por último, con las diferentes discusiones que aparecieron en la modernidad y en las reformas escolares, se plantea la necesidad de nombrar esos diferentes saberes bajo el nombre de "Ciencias de la Educación", en la medida en que estos saberes reclamaron el derecho a conceptualizar lo pedagógico. 


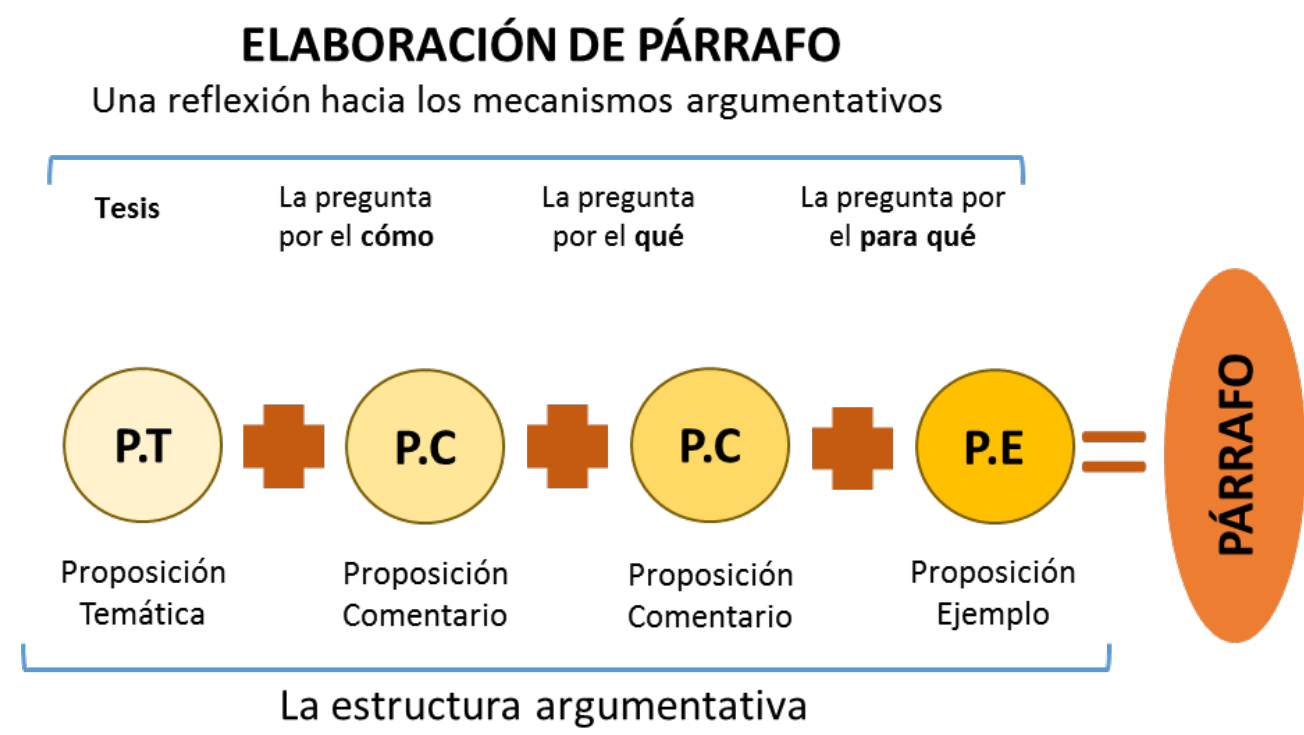

Fuente: Elaboración propia

La gráfica anterior muestra la estructura de un párrafo que contiene por sí mismo mecanismos argumentativos definidos como el qué, el cómo, el por qué y el para qué. Una vez se tenga el inventario de proposiciones, se pasa a reorganizar en forma lógico evidencial, las proposiciones en función de las razones para creer que la tesis es verdadera o falsa. Con estas argumentaciones se hace mucho más fácil estructurar el párrafo bajo mecanismos argumentativos sólidos.

\section{Tercer momento: más que conclusiones, puntos de partida}

\section{La síntesis y los acuerdos}

Este tercer momento es de síntesis de la discusión. Aquí se rescatan los principales argumentos y los principales acuerdos de la sesión. Vale la pena aclarar, que, como trabajo analítico y sintético, esta recuperación de los principales acuerdos, requiere recuperar la estructura argumentativa de los relatos, textos y otros dispositivos. Aquí lo más importante, es contar con unas formas más o menos claras de conectar las diferentes argumentaciones recolectadas.

\section{Últimas recomendaciones}

Se sugiere para la redacción de la reseña crítica utilizar el siguiente tiempo verbal: Tercera Persona del Presente Simple. Además, evitar el uso abusivo de gerundios y adverbios; en caso de usar gerundios, se recomienda utilizarlo después de un verbo. 


\section{Referencias para consultar}

Baquero V., J., \& Pardo P., J. (2017). Textos científicos y argumentativos. Una metodología para su comprensión y redacción. ÁNFORA, 5(10), 56-71. https://doi.org/https://doi.org/10.30854/anf.v5.n10.1997.369

Bolívar A. (2008) La didáctica como disciplina: historia y resignificación actual en: Didáctica y currículo de la modernidad a la postmodernidad, ediciones Aljibe, Málaga, España

Bourdieu, P. (2002) Las condiciones sociales de la circulación internacional de las ideas. En: Carolina Resoloagui, traductora cita original les conditions sociales de la circulation internalionale des idéss Actes de la reserche en sciencies sociales N 145, 2002

Bourdieu, P. (2008) ¿qué es hacer hablar a un autor? A propósito de Michel Foucault en: Capital cultural, escuela y espacio social, siglo veintiuno editores Madrid, España

Bourdieu, P., Chamboderon, J P., y Passeron, J. C., (2002), la construcción del objeto" en El oficio de sociólogo. Presupuestos epistemológicos, Siglo XXI, México.

Guédon, Jean-Claude (2011). El acceso abierto y la división entre ciencia "principal” y “periférica”. Crítica y Emancipación, (6): 135-180,

Llano, F. (2010) el sujeto objetivante en la investigación social en: Revista el educador Gran Colombiano N 8, Universidad La Gran Colombia, Bogotá, Colombia

Pardo Pardo, J. Felipe; Baquero Velásquez, Julia Marlén. La estructura argumentativa: base para la comprensión y producción de textos científicos y argumentativos. Forma y Función, [S.1.], n. 14, p. 98-118, ene. 2001. ISSN 2256-5469. Disponible en: $<$ https://revistas.unal.edu.co/index.php/formayfuncion/article/view/17218>. Fecha de acceso: 27 sep. 2018

Serna Dimas A (2015). Disertación elemental algunas cuestiones sobre la investigación social, Universidad Santo Tomas, Bogotá, Colombia 